\title{
ON THE RAMANUJAN CONJECTURE FOR QUASISPLIT GROUPS*
}

\author{
FREYDOON SHAHIDI ${ }^{\dagger}$
}

\author{
To the memory of Armand Borel
}

1. Introduction. Early experiences with classical (holomorphic) cusp forms, which initially started with the Ramanujan $\tau$-function, and later extended to even Maass cusp forms (cf. [70];[78], last paragraph) on the upper half plane, suggested that their Fourier coefficients $a_{p}$ at a prime $p$ must be bounded by $2 p^{(k-1) / 2}$, where $k$ is the weight (cf. $[25,96])$. This is what is classically called the Ramanujan-Petersson conjecture. Its archimedean counterpart, the Selberg conjecture [79], states that the positive eigenvalues of the hyperbolic Laplacian on the space of cuspidal functions (functions vanishing at all the cusps) on a hyperbolic Riemann surface parametrized by a congruence subgroup must all be at least $1 / 4$ (cf. [76, 79, 94]). While for the holomorphic modular cusp forms, this is a theorem ([25], also see $[8,12])$, the case of Maass forms is far from resolved and both conjectures are yet unsettled and out of reach.

Satake [78] was the first to observe that both conjectures can be uniformly formulated. More precisely, if one considers the global cuspidal representation attached to a given cuspidal eigenfunction, then all its local components must be tempered. This means that their matrix coefficients must all belong to $L^{2+\varepsilon}\left(P G L_{2}\left(\mathbb{Q}_{p}\right)\right)$ for all $\varepsilon>0$ and every prime $p$ of $\mathbb{Q}$. We note that here we are allowing $p=\infty$ and letting $\mathbb{Q}_{\infty}=\mathbb{R}$.

It is now generally believed that the conjecture in its general form should be valid for $G L_{n}$ over number fields to the effect that all the local components of an irreducible (unitary) cuspidal representation of $G L_{n}\left(\mathbb{A}_{F}\right)$ must be tempered (modulo center). Here $\mathbb{A}_{F}$ is the ring of adeles of our number field $F$. When $F$ is a function field (of one variable over a finite field), this is now a consequence of "purity" results of Lafforgue [58, 59], following the work of Drinfeld [26, 27] for $n=2$.

When $F$ is a number field, the conjecture is proved only in special cases, and only when the archimedean components are of geometric nature, i.e., that they can be realized in an appropriate cohomology [35], generalizing the holomorphic structure of classical modular forms, which are no longer available when $n \geq 3$; a characteristic missing from Maass forms which are only real analytic. In particular, the results in [35] require that $F$ be of $C M$-type. When $n=2$, generalizing the work of Deligne in the holomorphic case [25] to other number fields, has not been easy. Although significant progress is made in the case of Hilbert modular forms of weights $\geq 2$, $[8,12]$, the Ramanujan conjecture for a general holomorphic form of mixed weight 1 , i.e., where some of the weights are equal and some bigger than 1, is still unresolved.

As for other groups, the problem is even more complicated. In fact, one no longer expects that all the cuspidal representations of an arbitrary group are tempered, i.e., satisfy the Ramanujan conjecture; there are already counterexamples even for $S p_{4}$ (cf. [38, 52]). Consequently, in [64], Langlands formulates the conjecture in terms of functoriality (Sections 3 and 4, here) and as we explain in Section 5, functoriality then

\footnotetext{
*Received April 26, 2004; accepted for publication May 27, 2004.

${ }^{\dagger}$ Department of Mathematics, Purdue University, West Lafayette, IN 47907-2067, USA (shahidi@math.purdue.edu). The author was partially supported by NSF grant DMS-0200325.
} 
becomes a powerful tool in proving the conjecture for other groups by transferring the representation in question to an appropriate one on some $G L_{N}\left(\mathbb{A}_{F}\right)$. It is by means of functoriality that one can characterize those which do not satisfy the conjecture. We discuss these issues in Section 5, paragraph 5.2 (cf. the final remark in Section 10 of [21]).

It is through functoriality that one can obtain partial results towards the conjecture and in fact recent instances of functoriality proved for forms on different $G L_{N}$ 's has allowed us to obtain some striking estimates towards both the Ramanujan and Selberg conjectures for Maass forms [46, 49, 52, 53]. The fact that the cases of functoriality proved in $[46,51,53]$ are independent of whether the forms are holomorphic or not is of fundamental significance. We refer to [4, 17, 31, 37, 76, 77, 91, 92, 94] for some recent expository articles on these estimates and their consequences.

Another approach to the problem is that of "Automorphic Duals" due to BergerLi-Sarnak, which roughly speaking approaches the problem through understanding which local representations can possibly appear as a local component of an automorphic representation. Recent excellent articles by Clozel [17] and Sarnak [77] address these issues, and in particular [17] studies how the approach of automorphic duals can be mixed with Arthur's conjecture [2] in understanding non-tempered automorphic representations, to offer a better understanding of the Ramanujan conjecture. We refer the reader to $[17,77]$ since we will not discuss these issues here in our paper.

If one restricts oneself to quasisplit groups, i.e., those with a Borel subgroup defined over $F$, then there is a family of cuspidal representations which can be confidently conjectured to be tempered $([21,38,56,64,86]$, particularly Section 10 of [21]). These are the so called (globally) generic cuspidal representations [84, 86], i.e., those which have non-zero Fourier coefficients of highest rank (cf. Section 2). In this case and for many families of groups, functoriality is in a very good shape. In fact, it is the subject matter of $[20,21,48]$ that for almost all the quasisplit classical groups (including all the split ones) every generic cuspidal representation can be transferred through functoriality to an automorphic representation of an appropriate $G L_{N}\left(\mathbb{A}_{F}\right)$ (cf. Section 4 and 5). Consequently, the Ramanujan conjecture for them reduces to that of $G L_{N}$. Even without relying on the conjecture for $G L_{N}$, one can deduce new, and for the first time, global estimates towards the conjecture for these groups using those on $G L_{N}$ established in [69]. The extension to a quasisplit group whose connected $L$-group has a classical derived group [7] is discussed in paragraph 4.4.

We have surveyed the proofs in Section 6. It is here that we sketch the steps of the proof of functoriality in some of the cases discussed in Section 4. This is a difficult task as it involves two decades of development in the two major techniques involved, converse theorems of Cogdell and Piatetski-Shapiro and the LanglandsShahidi method on $L$-functions. We have concentrated our effort on only presenting the method in the cases of split connected reductive groups $\mathbf{G}$ for which ${ }^{L} G^{0}$ has a classical derived group, and have explained our method through them. We have remained very brief when dealing with establishing functoriality for the intriguing and important cases of $\operatorname{Sym}^{3}(\pi)$ and $\operatorname{Sym}^{4}(\pi)$, where $\pi$ is a cuspidal representation of $G L_{2}\left(\mathbb{A}_{F}\right)$ which were proved in $[46,53]$ and which relies on all the theory developed in the method [32, 45, 47, 83, 84, 85, 86, 87, 88] and more [5, 15, 41]. One reason has been the availability of some recent expositions of [31, 37, 76, 91, 92, 94]; particularly the very recent one [94] which has limited itself to the results on $G L_{2}$.

It is both with sadness and honor that I would like to dedicate this paper to the memory of Armand Borel. His keen interest in our recent work on functoriality 
prompted me to prepare this paper in his memory and in a rather general setting that he would have liked. I would like to thank the editors of the volume for their invitation.

2. Ramanujan Conjecture for Quasisplit Groups. Let $F$ be a global field (a number field or a function field of one variable over a finite field) and for each place $v$ of $F$, let $F_{v}$ be its completion at $v$. When $v$ is a discrete place, we let $\mathcal{O}_{v}$ be the ring of integers of $F_{v}$ and denote by $\mathcal{P}_{v}$ its maximal ideal. Let $q_{v}$ be the cardinality of the residue field $\mathcal{O}_{v} / \mathcal{P}_{v}$. Let $\varpi_{v}$ be a generator for $\mathcal{P}_{v}$ and fix an absolute value $\mid{ }_{v}$ such that $\left|\varpi_{v}\right|_{v}=q_{v}^{-1}$. Denote by $\mathbb{A}=\mathbb{A}_{F}$ the ring of adeles of $F$ and let $\mathbb{I}=\mathbb{I}_{F}=\mathbb{A}_{F}^{*}$ be the group of ideles. Finally, let $F_{s}$ be the separable closure of $F$ inside a fixed algebraic closure $\bar{F}$ of $F$ and set $\Gamma=\operatorname{Gal}\left(F_{s} / F\right)$.

Let $\mathbf{G}$ be a quasisplit connected reductive algebraic group over $F$. Fix a Borel subgroup $\mathbf{B}$ of $\mathbf{G}$ defined over $F$ and write $\mathbf{B}=\mathbf{T} \mathbf{U}$, where $\mathbf{T}$ is a maximal torus of $\mathbf{B}$ (maximally split torus of $\mathbf{G}$ ) and $\mathbf{U}$ its unipotent radical. We shall use $\mathbf{P}$ to denote a parabolic subgroup of $\mathbf{G}$ defined over $F$ which we will assume to contain $\mathbf{B}$. We fix a Levi decomposition $\mathbf{P}=\mathbf{M N}$ for which $\mathbf{T} \subset \mathbf{M}$. Note that $\mathbf{N} \subset \mathbf{U}$.

For each group $\mathbf{H}$ defined over $F$, we let $H_{v}=\mathbf{H}\left(F_{v}\right)$ and denote by $H=\mathbf{H}\left(\mathbb{A}_{F}\right)$, its adelic points. We then have $G, B, T, U, P, M, N$ as well as $G_{v}, B_{v}$, etc.

Let $\left\{X_{\alpha^{\prime}}\right\}$ be a set of simple root vectors, each defined for a simple root $\alpha^{\prime}$ of $\mathbf{T}$ in the Lie algebra of $\mathbf{U}$ and which are preserved under the action of $\Gamma$, i.e., a $F$-splitting (cf. $[10,55,93])$. It will then define a $F$-map

$$
\mathbf{U} \stackrel{\phi}{\longrightarrow} \Pi \mathbb{G}_{a}
$$

where the product is over all the simple roots of $\mathbf{T}$. It can be extended to $U=\mathbf{U}\left(\mathbb{A}_{F}\right)$ and each $U_{v}=\mathbf{U}\left(F_{v}\right)$. Let $\psi=\otimes_{v} \psi_{v}$ be a non-trivial (additive) complex character of $F \backslash \mathbb{A}_{F}$ and define a character $\chi$ of $U$ by

$$
\chi(u)=\psi\left(\sum_{\alpha^{\prime}} x_{\alpha^{\prime}}\right)
$$

where $\phi(u)=\left(x_{\alpha^{\prime}}\right)_{\alpha^{\prime}}$.

Next, let $\mathbf{A}_{\mathbf{G}}$ be the split component of the center $\mathbf{Z}_{\mathbf{G}}$ of $\mathbf{G}$, i.e., the largest split subtorus of the connected component $\mathbf{Z}_{\mathbf{G}}^{o}$ of $\mathbf{Z}_{\mathbf{G}}$. Fix a (unitary) character $\rho$ of $\mathbf{A}_{\mathbf{G}}(F) \backslash A_{G}, A_{G}=\mathbf{A}_{\mathbf{G}}\left(\mathbb{A}_{F}\right)$, and let $\pi=\otimes_{v} \pi_{v}$ be a cuspidal subrepresentation of $L^{2}\left(A_{G} \mathbf{G}(F) \backslash G, \rho\right)$, i.e., those (irreducible) cuspidal representations which transform according to $\rho$ under the action of $A_{G}$.

The representation $\pi$ is called globally $\chi-$ generic, if there exists a cuspidal function $\varphi$ in its space for which

$$
\int_{\mathbf{U}(F) \backslash U} \varphi(u g) \overline{\chi(u)} d u \neq 0
$$

for some $g \in G$. Recall that $\mathbf{U}(F) \backslash U$ is compact.

When $\mathbf{G}=G L_{n}$, it is expected that cuspidal representations, which are all automatically globally generic [95], are tempered, i.e., for $\pi=\otimes_{v} \pi_{v}$ each $\pi_{v}$ is tempered. When $F$ is a function field this is in fact a theorem due to Lafforgue [58, 59] (cf. [26, 27] for $n=2$ ). But for number fields this is not known even for $n=2$.

On the other hand, in general this is false. In fact, there are counterexamples even for $S p_{4}\left(\mathbb{A}_{F}\right)$, i.e., arbitrary cuspidal representations of a general reductive group 
need not be tempered $[2,38,56]$. If a cuspidal representation $\pi$ is tempered, we will then say that $\pi$ satisfies the (generalized) Ramanujan conjecture as formulated first in [78] (also cf. [61, 64]). This is a very deep conjecture and even for $G L_{2}\left(\mathbb{A}_{F}\right)$, where $F$ is a number field, is unresolved. When $F=\mathbb{Q}$ and $\pi$ corresponds to a Maass form $[39,70,76,77]$ the temperedness of $\pi_{\infty}$ is equivalent to Selberg's conjecture, a celebrated conjecture $[79,94]$ demanding that there be no positive eigenvalues for Laplacian on any hyperbolic Riemann surface whose value is smaller than $1 / 4$. On the other hand, for those $\pi$ which correspond to holomorphic modular forms, the conjecture is a theorem proved by Deligne [25] as a consequence of his proof of Weil conjecture. For an arbitrary $F$, the results are incomplete even for $G L_{2}\left(\mathbb{A}_{F}\right)$ (cf. [8, $12,35])$.

For an arbitrary quasisplit group $\mathbf{G}$ and $\pi$ any globally generic cuspidal representation of $G=\mathbf{G}\left(A_{F}\right)$ one expects the following conjecture (cf. $[21,74,86]$ ).

Conjecture 2.1. Let $\pi=\otimes_{v} \pi_{v}$ be a globally generic cuspidal representation of $G$, the $\mathbb{A}_{F}$-points of a quasisplit connected reductive $F$-group $\mathbf{G}$. Then $\pi$ satisfies the Ramanujan conjecture, i.e., each $\pi_{v}$ is tempered. In particular, if $\mathbf{G}=G L_{n}$, then every cuspidal representation of $G L_{n}\left(\mathbb{A}_{F}\right)$ is tempered.

In this paper we will explain how the conjecture for one group can be reduced to another. This is done through another conjecture, that of Functoriality due to Langlands, in which there has been some remarkable recent progress. In particular, while we are still far away from a proof of Conjecture 2.1, this leads to new estimates towards temperedness of local components of globally generic cuspidal representations of many groups. In the case of $G L_{2}\left(\mathbb{A}_{F}\right)$, this provides us with some very surprising estimates $[46,49,52,53]$ towards Hecke eigenvalues of local components of a cusp form on $G L_{2}\left(\mathbb{A}_{F}\right)$ (automatically globally generic). When $F=\mathbb{Q}$, one gets

$$
\lambda_{1} \geq \frac{975}{4096} \simeq 0.2380371
$$

for positive eigenvalues of Laplacian

$$
\Delta=-y^{2}\left(\frac{\partial^{2}}{\partial x^{2}}+\frac{\partial^{2}}{\partial y^{2}}\right)
$$

on every hyperbolic Riemann surface parametrized by a congruence subgroup [39]. The conjectured lower bound (Selberg [79]) is $1 / 4=0.25$.

3. Functoriality Conjecture. Let $\mathbf{G}$ be as in Section 2, i.e., a quasisplit connected reductive algebraic group over $F$. Assume there exists a $L$-homomorphism

$$
\rho:{ }^{L} G \longrightarrow G L_{N}(\mathbb{C}) \times W_{F}^{\prime}
$$

for some positive integer $N$, where $W_{F}^{\prime}=W^{\prime}\left(F_{s} / F\right)$ is the corresponding DeligneWeil group (cf. $[9,99]$ ) and ${ }^{L} G={ }^{L} G^{\circ} \rtimes W_{F}^{\prime}$ is the $L$-group of $\mathbf{G}$. (We will define the "global" Weil-Deligne group by just adding a $S L_{2}(\mathbb{C})$-factor to $W_{F}$. This will be necessary for the discussion in Remark 3.2.) We refer to [9] for the definition of ${ }^{L} G$. Here, by an $L$-homomorphism we shall mean one satisfying $\rho(1 \rtimes w)=a(w) \times w$, for all $w \in W_{F}^{\prime}$, where $a(w) \in G L_{N}(\mathbb{C})$ depends on $w$.

If $W_{F_{v}}^{\prime}$ is the local Weil-Deligne group at a place $v$, then the natural map $W_{F_{v}}^{\prime} \longrightarrow$ $W_{F}^{\prime}$ implies a natural homomorphism $\eta_{v}:{ }^{L} G_{v}={ }^{L} G^{\circ} \rtimes W_{F_{v}}^{\prime} \longrightarrow{ }^{L} G$. The map $\rho \cdot \eta_{v}$ is then a $L$-homomorphism from ${ }^{L} G_{v}$ into $G L_{N}(\mathbb{C}) \times W_{F_{v}}^{\prime}$. 
Let $\pi=\otimes_{v} \pi_{v}$ be an automorphic representation of $G$. For almost all places $v$, where $\mathbf{G}$ is unramified, i.e., splits over an unramified extension, the class of representation $\pi_{v}$ is uniquely determined by a semisimple conjugacy class $t_{v}$ in ${ }^{L} G_{v}$ (cf. $[9,61])$. The image of $\left(\rho \cdot \eta_{v}\right)\left(t_{v}\right)$ then generates a semisimple conjugacy class in $G L_{N}(\mathbb{C}) \times W_{F_{v}}^{\prime}$. Let $\Pi_{v}$ be the unramified representation of $G L_{N}\left(F_{v}\right)$ attached to this class. Langlands functoriality conjecture in this setting can be formulated as follows.

ConjeCtuRe (LANGLANDS $[4,61]) 3.1$. There exists an automorphic representation $\Pi^{\prime}=\otimes \Pi_{v}^{\prime}$ of $G L_{N}\left(\mathbb{A}_{F}\right)$ such that $\Pi_{v}^{\prime}=\Pi_{v}$ for all $v \notin S$, where $S$ is a finite set of places for which $v \notin S$ implies that both $\mathbf{G}$ as a group over $F_{v}$ and $\pi_{v}$ are unramified.

REMARK 3.2. It is conjectured that every irreducible admissible representation $\pi_{v}$ (not necessarily a component of an automorphic form) of $G_{v}$ is parametrized by a homomorphism $\theta_{v}: W_{F_{v}}^{\prime} \longrightarrow{ }^{L} G_{v}$ in a natural way (cf. [9, 99]). This is what is sometimes called the Local Langlands Conjecture. One can then consider

$$
\rho \cdot \theta_{v}: W_{F_{v}}^{\prime} \longrightarrow G L_{N}(\mathbb{C}) \times W_{F_{v}}^{\prime}
$$

and let $\Pi_{v}$ be the representation of $G L_{N}\left(F_{v}\right)$ attached to $\rho \cdot \theta_{v}$ (cf. $\left.[35,36,65]\right)$. The Functoriality conjecture can then be reformulated to take the stronger form that:

Conjecture (Strong Functoriality Conjecture) 3.3. The representation $\Pi=\otimes_{v} \Pi_{v}$ is an automorphic representation of $G L_{N}\left(\mathbb{A}_{F}\right)$.

When $F$ is a number field and $v$ is an archimedean place, the local parametrization problem was completely resolved by Langlands in [65]. For $\mathbf{G}=G L_{N}$, the breakthrough was accomplished by Harris-Taylor [35] and independently by Henniart [36], a few years ago. In the case of $\mathbf{G}=S O_{2 n+1}$, the special split odd orthogonal group of rank $n$ (in $(2 n+1)$-variables) this has been recently established by Jiang and Soudry [44], using certain cases of functoriality [20] and descent [33, 98] to reduce it to the case of $G L_{N}$ and thus using $[35,36]$.

4. Recent Instances of Functoriality. There are certain instances of functoriality that have been established recently. Some were proved a few years ago $[20,46,51,53]$. But some others are quite new and still in preprint form [7, 21, 48]. In this section we will explain what they are and later (Section 6) give a brief sketch of how they are proved. We take up their consequences towards the Ramanujan conjecture in the next chapter.

We start with certain cases involving $G L_{n}$, the general linear group.

4.1. Products for $G L_{n}$. Let $n_{1}$ and $n_{2}$ be two positive integers. Given any positive integer $N$, it is well-known that ${ }^{L} G L_{N}=G L_{N}(\mathbb{C}) \times W_{F}^{\prime}$. Let $\mathbf{G}=G L_{n_{1}} \times$ $G L_{n_{2}}$ and let

$$
\rho: G L_{n_{1}}(\mathbb{C}) \times G L_{n_{2}}(\mathbb{C})={ }^{L}\left(G L_{n_{1}} \times G L_{n_{2}}\right)^{o} \longrightarrow G L_{n_{1} n_{2}}(\mathbb{C})
$$

be the map $\rho\left(g_{1}, g_{2}\right)=g_{1} \otimes g_{2}$. Here since the groups are split, we have dropped the group $W_{F}^{\prime}$ and confined ourselves to the connected component ${ }^{L} G^{o}$ of ${ }^{L} G$. No generality will be lost.

Functoriality for $\rho$ is now proved for $n_{1}=n_{2}=2([46,75])$ and $n_{1}=2$ and $n_{2}=3$ $([51,53])$. (The cases where one or both $n_{1}$ and $n_{2}$ are equal to 1 are trivial.) The 
results proved in $[46,51,53,75]$ are in fact that of functoriality in its strongest form, i.e., Conjecture 3.3. Beyond these cases, there are no other pairs $n_{1}$ and $n_{2}$ for which even Conjecture 3.1 is proved. Of course, as pointed out earlier, the local situation is quite different and the local Langlands functoriality has now been established for any $n_{1}$ and $n_{2}$, and at all the places. The case of archimedean places is due to Langlands [65], while the non-archimedean ones were established in [35, 36]. Thus given two cuspidal representations $\pi_{1}=\otimes_{v} \pi_{1 v}$ and $\pi_{2}=\otimes_{v} \pi_{2 v}$ of $G L_{n_{1}}\left(\mathbb{A}_{F}\right)$ and $G L_{n_{2}}\left(\mathbb{A}_{F}\right)$, let $\pi_{1 v} \otimes \pi_{2 v}$ be the representation of $G L_{n_{1} n_{2}}\left(\mathbb{A}_{F}\right)$ attached to $\theta_{1 v} \otimes \theta_{2 v}$, where

$$
\theta_{i v}: W_{F_{v}}^{\prime} \longrightarrow G L_{n_{i}}(\mathbb{C}) \quad(i=1,2)
$$

is the parametrization of $\pi_{i v}$ as in [35, 36, 65] (cf. Remark 3.2). The representation $\Pi_{v}$ in Conjecture 3.3 must be $\Pi_{v}=\pi_{1 v} \otimes \pi_{2 v}$ and the content of $[46,51,53,75]$ is that in the cases $n_{1}=n_{2}=2$ and $n_{1}=2, n_{2}=3$ this is indeed the case. One sets $\pi_{1} \otimes \pi_{2}=\otimes_{v}\left(\pi_{1 v} \otimes \pi_{2 v}\right)$.

This case of functoriality is very important and as pointed out by Langlands [64], although much weaker, reflects the validity of global Langlands correspondence for $G L_{n}$ over number fields, a matter which is now a theorem due to Lafforgue in the case of function fields $[58,59]$ and which has this functoriality as one of its corollaries [59].

4.2. Symmetric powers for $G L_{2}$. Let $m$ be a positive integer. Let $P(x, y)$ be a homogeneous polynomial of degree $m$ in two variables $x$ and $y$. The change of variables $(x, y) \mapsto(x, y) g, g \in G L_{2}(\mathbb{C})$, changes the coefficients in $P(x, y)$ through multiplication by a matrix $\operatorname{Sym}^{m} g \in G L_{m+1}(\mathbb{C})$. The map

$$
\operatorname{Sym}^{m}: G L_{2}(\mathbb{C}) \longrightarrow G L_{m+1}(\mathbb{C})
$$

is a homomorphism and the proof of functoriality for $\mathrm{Sym}^{m}$ is one of the most interesting and important problems in automorphic forms partly due to its number theoretic and arithmetic geometric applications. In fact, if established for all $m$ it proves both the Ramanujan and Selberg conjectures for forms on $G L_{2}$ at once. It will also immediately imply the Sato-Tate conjecture on equidistribution of Hecke eigenvalues of these forms $[52,80,81]$.

While again only for a local field is this established for all $m$, there has recently been tremendous progress in establishing functoriality for $\mathrm{Sym}^{m}$ over number fields. While the case of $m=2$ was established in [30] some 25 years ago, the cases $m=3$ (cf. [51, 53]) and $m=4([46])$ are quite recent. Again in all the cases functoriality is now proved in the strong form of Conjecture 3.3, i.e., that

$$
\operatorname{Sym}^{m} \pi=\otimes_{v} \operatorname{Sym}^{m} \pi_{v}
$$

is an automorphic representation of $G L_{m+1}\left(\mathbb{A}_{F}\right)$, for $m=2,3$ and 4 . Here $\operatorname{Sym}^{m} \pi_{v}$ is the representation of $G L_{m+1}\left(F_{v}\right)$ attached to (parametrized by) $\operatorname{Sym}^{m} \theta_{v}$, where

$$
\theta_{v}: W_{F_{v}}^{\prime} \longrightarrow G L_{2}(\mathbb{C})
$$

is the corresponding parametrization of $\pi_{v}$ and $\operatorname{Sym}^{m} \theta_{v}=\mathrm{Sym}^{m} \cdot \theta_{v}$.

The automorphy of $\mathrm{Sym}^{3} \pi$ has been a long standing problem $[14,50,62,82]$ and while historically attempts had been made to establish functoriality of $\mathrm{Sym}^{3}$ directly, it finally became a consequence of the functoriality of $\pi_{1} \otimes \pi_{2}$ discussed in 4.1 applied to $\pi_{1}=\pi$ and $\pi_{2}=S y m^{2} \pi$. In fact,

$$
\pi \otimes S y m^{2} \pi=S y m^{3} \pi \boxplus\left(\pi \otimes \omega_{\pi}\right),
$$


i.e., that the automorphic representation $\pi \otimes S y m^{2} \pi$ is defined by means of the representation of $G L_{6}\left(\mathbb{A}_{F}\right)$ induced from the representation $\left(\operatorname{Sym}^{3} \pi, \pi \otimes \omega_{\pi}\right)$ of $G L_{4}\left(\mathbb{A}_{F}\right) \times G L_{2}\left(\mathbb{A}_{F}\right)$. This is done by means of incomplete (or pseudo-) Eisenstein series. This is simply an inverse Mellin transform of the corresponding Eisenstein series. We refer to [39], page 57, for this particular terminology and to [71] for "pseudo-Eisenstein series" (cf. also [1,63]). In particular, $S y m^{3} \pi$ is automorphic [43]. Here $\omega_{\pi}$ is the central character of $\pi$.

The proof of automorphy of $\mathrm{Sym}^{4} \pi$ is again indirect. One first proves the functoriality for the exterior square map

$$
\Lambda^{2}: G L_{4}(\mathbb{C}) \longrightarrow G L_{6}(\mathbb{C}),
$$

attaching to every cuspidal representation $\Pi$ of $G L_{4}\left(\mathbb{A}_{F}\right)$ an automorphic representation of $G L_{6}\left(\mathbb{A}_{F}\right)$ as it was done by Kim in [46]. One then uses

$$
\Lambda^{2}\left(\operatorname{Sym}^{3} \pi\right)=\left(\operatorname{Sym}^{4} \pi \otimes \omega_{\pi}\right) \boxplus \omega_{\pi}^{3}
$$

to conclude that $\operatorname{Sym}^{4} \pi$ is an automorphic representation of $G L_{5}\left(\mathbb{A}_{F}\right)$ as in the previous case.

4.3. Classical Groups. By a classical group we shall mean a connected reductive algebraic group fixing either a symmetric, alternating, or hermitian form. Here we shall assume $\mathbf{G}$ is a quasisplit classical group and consider the natural embedding

$$
i:{ }^{L} G \longrightarrow G L_{N}(\mathbb{C}) \times W_{F}^{\prime} .
$$

We will assume $N$ is minimal. Then, if $\mathbf{G}=S O_{m}$ and $n=[m / 2]$, then $N=2 n$. On the other hand for $\mathbf{G}=S p_{2 n}, N=2 n+1$, while for $\mathbf{G}=U(n, n)$ or $\mathbf{G}=$ $U(n+1, n), N=2 n$ or $2 n+1$, respectively. The action of $\Gamma$ on $G L_{N}(\mathbb{C})$ is always trivial, its action on ${ }^{L} G^{o}$ is either trivial or through the defining extension when $\mathbf{G}=U(n, n), U(n+1, n)$, or $S O_{2 n}^{*}$, a quasisplit form of $S O_{2 n}$ (cf. [3]). The action of $\Gamma$ on ${ }^{L} G^{o}$ dualizes that on $\mathbf{G}$ (cf. [9]).

For split classical groups the automorphy of $i$ on their globally generic spectrum has been established very recently in [21]. We refer to [20] for the case of $S O_{2 n+1}$ established earlier. For serious technical reasons [23] had to be postponed until [93] was written. More precisely, one needed to prove that certain local root numbers are stable and basically do not change if $\pi_{v}$ is twisted by a highly ramified character of $F_{v}^{*}$ (cf. $[23,21,93]$ ). The case of $U(n, n)$ is now proved in [48] and one expects little problem in extending it to $U(n+1, n)$. The case of quasisplit $S O_{2 n}$ which still needs to be treated will be taken up by the authors of $[20,21]$.

4.4. Groups whose connected $L$-groups have a classical derived group. The groups discussed in 4.3 are examples of groups which while themselves may not be classical, the connected components of their $L$-groups have a classical derived group. To complete the list one needs to consider similitude spin groups. We will restrict ourselves to split cases although the non-split ones should also be considered. In the split case these groups are defined (cf. [6]) to be

$$
\mathbf{G}=\operatorname{GSpin}_{m}=\left(G L_{1} \times \operatorname{Spin}_{m}\right) /\{(1,1),(-1, c)\},
$$

$c=\alpha_{n}^{\vee}(-1)$ if $m=2 N+1$ or $c=\alpha_{n-1}^{\vee}(-1) \alpha_{n}^{\vee}(-1)$ if $m=2 n$. Here $\operatorname{Spin}_{m}$ is the simply connected covering of $S O_{m}$ and $\alpha_{i}^{\vee}$ is the $i$-th coroot of $\operatorname{Spin}_{m}$. Note that 
${ }^{L} G^{o}=G S O_{2 n}$ if $m=2 n$ and ${ }^{L} G^{o}=G S p_{2 n}$ if $m=2 n+1$ and consequently in both cases

$$
i:{ }^{L} G \hookrightarrow G L_{2 n}(\mathbb{C}) \times W_{F}^{\prime} .
$$

Functoriality in the sense of Conjecture 3.1 has now been established for $i$ in [7].

4.5. The strong lift. In the cases discussed in 4.3 and 4.4, the local Langlands conjecture is not á priori available. Using the results of Harris-Taylor [35] and Henniart [36] one can reduce the local reciprocity (parametrization) for these groups to that for $G L_{n}$ if one uses the corresponding cases of functoriality proved in [7, 20,21,48] and appropriate descents [33, 98], i.e., an appropriate inverse to functoriality. While the descent has now been established for all the classical groups [33, 98], the case of GSpin groups is still not available. The descent for classical groups basically allows one to start with a self-dual cuspidal representation on $G L_{N}\left(\mathbb{A}_{F}\right)$ and descend it to a globally generic form on one of the quasisplit classical groups discussed in 4.3 (cf. [20, 21, 48]). For the case of GSpin-groups the descent should start with representations $\Pi$ of $G L_{2 n}\left(\mathbb{A}_{F}\right)$ for which $\Pi \simeq \tilde{\Pi} \otimes \eta$ for a grössencharacter $\eta$. Every cuspidal representation $\pi$ of a $G \operatorname{Spin}_{m}^{*}\left(\mathbb{A}_{F}\right)$ must transfer to a $\Pi$ and $\eta$ such that $\eta=\omega_{\pi}$ and $\omega_{\Pi} / \omega_{\pi}^{n}$ is equal to the quadratic character whose defining extension through class field theory is the defining extension for the quasisplit group $G S p_{2 n}^{*}$. For the split $G S p_{2 n}$ or $G S p_{2 n+1}$, this can be interpreted as $\omega_{\Pi}=\omega_{\pi}^{n}$. It should be remarked that if $\Pi$ is a representation of $G L_{2 n+1}\left(\mathbb{A}_{F}\right)$ satisfying $\Pi \simeq \tilde{\Pi} \otimes \eta$, then $\eta=\nu^{2}$ for another character $\nu$ and $\Pi_{1} \simeq \tilde{\Pi}_{1}$, where $\Pi_{1}=\Pi \otimes \nu^{-1}$. We like to conclude by pointing out that transfers from Gspin-groups to $G L_{N}$ are examples of the most general cases of twisted endoscopy, as developed by Kottwitz, Langlands and Shelstad [55, 67]. Such transfers are expected to be established by means of Arthur's twisted trace formula for all the automorphic representations. At present this requires the validity of appropriate fundamental lemmas which are still unavailable. We will discuss this in more detail later in Section 6, Remark 6.2.

\section{Applications to the Ramanujan Conjecture.}

5.1. $G L_{2}$ and $G L_{n}$. Some striking estimates are now established towards this conjecture when $G=G L_{2}\left(\mathbb{A}_{F}\right)$, where $F$ is a number field. (In the case of function fields the full conjecture is known to be true as a consequence of the resolution of parametrization problem for $G L_{n}\left(\mathbb{A}_{F}\right), F$ a function field, due to Drinfeld [26, 27] for $n=2$, and Lafforgue $[58,59]$ for any $n$. The conjecture is a consequence of purity for the parametrizing representations.) We shall now describe these estimates.

Let $\pi=\otimes_{v} \pi_{v}$ be a cuspidal representation of $G L_{2}\left(\mathbb{A}_{F}\right)$, where $F$ is a number field. For the unramified places $v$,

$$
t_{v}=\left(\begin{array}{cc}
\alpha_{v} & 0 \\
0 & \beta_{v}
\end{array}\right) \in G L_{2}(\mathbb{C})
$$

and the Ramanujan conjecture demands that

$$
\left|\alpha_{v}\right|=\left|\beta_{v}\right|=1 \text {. }
$$

In the generality of any number field, the best estimate is now proved in [52] as

THEOREM 5.1 [52]. Let $\pi=\otimes_{v} \pi_{v}$ be a cuspidal representation of $G L_{2}\left(\mathbb{A}_{F}\right)$. Then for all the unramified places

$$
q_{v}^{-1 / 9}<\left|\alpha_{v}\right|,\left|\beta_{v}\right|<q_{v}^{1 / 9} .
$$


A similar result to this is also proved for archimedean places in [47].

When $F=\mathbb{Q}$, better estimates are available due to Kim and Sarnak:

TheOREm 5.2 [49]. Assume $F=\mathbb{Q}$ and let $\pi=\otimes_{p} \pi_{p}$ be a cusp form on $G L_{2}\left(\mathbb{A}_{\mathbb{Q}}\right)$. Let $p$ be a finite prime at which $\pi_{p}$ is unramified and use $t_{p}=\operatorname{diag}\left(\alpha_{p}, \beta_{p}\right) \in$ $G L_{2}(\mathbb{C})$ to denote the attached semisimple element. Then

$$
p^{-7 / 64} \leq\left|\alpha_{p}\right|,\left|\beta_{p}\right| \leq p^{7 / 64} .
$$

It is important to recall the connection of these estimates to the historically important cases of classical modular forms and Maass forms on the upper half plane where the Ramanujan conjecture was first stated.

It is well known that each weight $k$ holomorphic modular or Maass cusp form $f$ on the upper half plane which is an eigenfunction for all the Hecke operators as well as the Laplacian corresponds to a cuspidal representation $\pi=\pi_{f}=\otimes_{p} \pi_{p}$ of $G L_{2}\left(\mathbb{A}_{\mathbb{Q}}\right)$ (cf. [29] for example). If we further normalize $f$ so that its first Fourier coefficient $a_{1}=1$, then the Ramanujan conjecture is equivalent to

$$
\left|a_{p}\right| \leq 2 p^{(k-1) / 2}
$$

where $a_{p}$ is the Fourier coefficient at a prime $p$ which does not divide the level of $f$. In the case of Maass forms $k=0$ and the conjecture asserts

$$
\left|a_{p}\right| \leq 2 p^{-1 / 2}
$$

In fact, it is easy to see that

$$
a_{p}=p^{\frac{k-1}{2}}\left(\alpha_{p}+\beta_{p}\right),
$$

where $\operatorname{diag}\left(\alpha_{p}, \beta_{p}\right)$ parametrizes $\pi_{p}$, from which one can immediately deduce the equivalence of (5.1.2) and (5.1.5) since $a_{p} \in \mathbb{R}$.

For the holomorphic forms the conjecture was proved by Deligne [25] in 1973, as a consequence of his proof of Weil conjecture. For Maass forms the problem is open and the geometric techniques which were useful in the holomorphic form due to the holomorphic structure renders useless, as these forms are only real analytic.

As for the place at infinity, i.e., $\mathbb{Q}_{\infty}=\mathbb{R}$, the problem is equivalent to that of Selberg [79]. In fact, for a holomorphic cusp form of weight $k, \pi_{\infty}$ will be the discrete series of lowest weight $k$ and automatically tempered. On the other hand for a Maass cusp form, the story is very different. Here $\pi_{\infty}$ is a representation fully induced from a pair of characters $\mu_{\infty}$ and $\nu_{\infty}$ of $\mathbb{R}^{*}$ with

$$
\mu_{\infty} / \nu_{\infty}=||^{s_{\infty}}
$$

$s_{\infty} \in \mathbb{C}$. Let $\Gamma$ be the congruence subgroup attached to $f$ and let $\lambda_{1}=\lambda_{1}(\Gamma \backslash H)$ be the smallest positive eigenvalue for

$$
\Delta=-y^{2}\left(\frac{\partial^{2}}{\partial x^{2}}+\frac{\partial^{2}}{\partial y^{2}}\right)
$$

on $L^{2}(\Gamma \backslash H)$, where $H$ denotes the upper half plane. Let $\lambda$ be the eigenvalue of $\Delta$ at $f$, i.e., $\Delta f=\lambda f$. Then $\lambda=\frac{1-s_{\infty}^{2}}{4}>0$ and $\lambda \geq \lambda_{1}$. The Selberg conjecture [79] requires: 
Conjecture 5.3 (SElBerg [79]). $\lambda_{1} \geq 1 / 4$ or equivalently $s_{\infty} \in i \mathbb{R}$. Observe that this is equivalent to $\mu_{\infty}$ and $\nu_{\infty}$ being unitary characters and thus $\pi_{\infty}$ is tempered.

The best estimate towards the Selberg conjecture is again due to Kim-Sarnak [49]:

THEOREM $5.4[49] . s_{\infty} \in[-7 / 32,7 / 32] \cup i \mathbb{R}$ or equivalently $\lambda \geq \lambda_{1} \geq \frac{1}{4}-\left(\frac{7}{64}\right)^{2}=$ $\frac{975}{4096} \simeq 0.2380371$.

When $F$ is arbitrary, the best way to state the result at an archimedean place is to use $s_{v}$ defined by

$$
\mu_{v} / \nu_{v}=||_{v}^{s_{v}} \quad\left(s_{v} \in \mathbb{C}\right)
$$

where $v$ is an archimedean place of $F$. In fact, suppose $\pi_{v}=I\left(\mu_{v}, \nu_{v}\right)$ and assume

$$
\mu_{v}=||_{v}^{s_{1 v}}
$$

and

$$
\nu_{v}=|| \begin{aligned}
& s_{2 v} \\
& v
\end{aligned}
$$

and therefore $s_{v}=s_{1 v}-s_{2 v}$. Then $\operatorname{Re}\left(s_{1 v}\right)+\operatorname{Re}\left(s_{2 v}\right)=0$, while $\operatorname{Re}\left(s_{v}\right)=\operatorname{Re}\left(s_{1 v}\right)-$ $\operatorname{Re}\left(s_{2 v}\right)=2 \operatorname{Re}\left(s_{1 v}\right)$. When $F=\mathbb{Q}, s_{v}=s_{\infty}$ defined by (5.8) and therefore the Ramanujan-Selberg conjecture at every archimedean place requires that

$$
\operatorname{Re}\left(s_{v}\right)=\operatorname{Re}\left(s_{i v}\right)=0 .
$$

Then the best present estimate which is stated in [47] can be written as:

ThEOREM 5.5 [47]. $\left|R e\left(s_{j v}\right)\right| \leq 1 / 9, j=1,2$, at every archimedean place $v$ of F. Equivalently $\left|\operatorname{Re}\left(s_{v}\right)\right| \leq 2 / 9$.

We refer to [94] for a recent exposition of these issues and more details viz-a-viz holomorphic modular forms and Maass forms. Among other surveys of these problems we like to refer to recent expositions in $[18,37,76,77,91]$.

These results are proved by using functorial transfers $S y m^{3} \pi$ and $S y m^{4} \pi$ discussed in paragraph 4.2, together with techniques from analytic number theory [13, 68] or those developed in [86]. In fact, having $S y m^{3} \pi$ and $S y m^{4} \pi$ as automorphic forms on $G L_{4}\left(\mathbb{A}_{F}\right)$ and $G L_{5}\left(\mathbb{A}_{F}\right)$, respectively, one can always use the general global bounds of Luo-Rudnick-Sarnak [69] for cusp forms on $G L_{n}\left(\mathbb{A}_{F}\right)$ to improve on $\left|\alpha_{v}\right|$ and $\left|\beta_{v}\right|$ by bounding $\left|\alpha_{v}^{m}\right|$ and $\left|\beta_{v}^{m}\right|$ by

$$
q_{v}^{-\left(\frac{1}{2}-\frac{1}{n^{2}+1}\right)} \leq\left|\alpha_{v}\right|^{m},\left|\beta_{v}\right|^{m} \leq q_{v}^{\frac{1}{2}-\frac{1}{n^{2}+1}}
$$

with $n=m+1$. Here we can take $m=3$ and 4 leading to

$$
q_{v}^{-5 / 34} \leq\left|\alpha_{v}\right|,\left|\beta_{v}\right| \leq q_{v}^{5 / 34}
$$

using $m=3$ (cf. [53]) and

$$
q_{v}^{-3 / 26} \leq\left|\alpha_{v}\right|,\left|\beta_{v}\right| \leq q_{v}^{3 / 26}
$$

using $m=4$ (cf. [46]) with similar bounds at archimedean places. Although, neither results are as strong as those in Theorems $5.1,5.2,5.4$ or 5.5 , they were much stronger than what was available before them $[13,68,86]$. 
The estimates in [69] are the best available for cuspidal representations of $G L_{n}\left(\mathbb{A}_{F}\right)$. They are non-local and therefore a breakthrough. They are also the best concrete evidence so far towards the Ramanujan conjecture for $G L_{n}\left(\mathbb{A}_{F}\right)$ and the source of allowing us to make gains towards the conjecture for other groups using functoriality as we explain below.

5.2. Classical groups. We will continue to assume that $F$ is a number field. As explained earlier Ramanujan's conjecture is not valid for arbitrary cusp forms on even split classical groups. This is evident from examples of Howe-Piatetski-Shapiro [38] and Kurokawa [56] already for $S p_{4}$. To remedy this, in [64] Langlands suggested that if, for example, $\mathbf{G}$ is a classical group with a minimal embedding

$$
i:{ }^{L} G \hookrightarrow G L_{N}(\mathbb{C}) \times W_{F}^{\prime},
$$

then a cuspidal representation $\pi=\otimes_{v} \pi_{v}$ of $G=\mathbf{G}\left(\mathbb{A}_{F}\right)$ which defies the Ramanujan conjecture, can not be transferred functorially to an "isobaric" representation of $G L_{N}\left(\mathbb{A}_{F}\right)$ (cf. [43]). Roughly speaking, an isobaric representation is one whose local components are all Langlands quotients of the corresponding inducing representations given by the global automorphic data [11, 65, 97].

More precisely, if $\Pi_{1} \otimes \Pi_{2} \otimes \ldots \otimes \Pi_{r}$ is the inducing cuspidal data for an automorphic representation $\Pi$ of $G L_{N}\left(\mathbb{A}_{F}\right)$ in which $\Pi_{i}=\Pi_{i 0} \otimes|\operatorname{det}|^{s_{i}}, 1 \leq i \leq r$, where each $\Pi_{i 0}$ is unitary cuspidal and $s_{i} \in \mathbb{R}$, with $s_{1} \geq s_{2} \geq \ldots \geq s_{r}$, one then considers representations induced from $\Pi_{1 v} \otimes \ldots \otimes \Pi_{r v}$ for each $v$. Writing each $\Pi_{i v}$ as the Langlands quotient of the corresponding standard module and further rearranging the corresponding Langlands data $[11,65,97]$ if necessary, one can conclude that each representation induced from $\Pi_{1 v} \otimes \ldots \otimes \Pi_{r v}$ has a unique quotient which we denote by $\Pi_{1 v} \boxplus \ldots \boxplus \Pi_{r v}$. No rearrangement is necessary if $\Pi_{i v}$ 's are all tempered which is expected to be the case by the Ramanujan conjecture for $G L_{m}\left(\mathbb{A}_{F}\right)$. One then sets $\Pi_{1} \boxplus \ldots \boxplus \Pi_{r}$ for the tensor product of all the $\Pi_{1 v} \boxplus \ldots \boxplus \Pi_{r v}$ which is a constituent of the representation induced from $\Pi_{1} \otimes \Pi_{2} \ldots \otimes \Pi_{r}$. This is what we call an "isobaric" representation of $G L_{N}\left(\mathbb{A}_{F}\right)$. If each $s_{i}=0$, we then call $\Pi_{1} \boxplus \ldots \boxplus \Pi_{r}$ "unitary isobaric".

One of the important results of $[21,48]$ is that:

TheOrem $5.6[21,48]$. Let $\pi=\otimes_{v} \pi_{v}$ be a globally generic cuspidal representation of eiher a split classical group $G=\mathbf{G}\left(\mathbb{A}_{F}\right)$ or a unitary group $U(n, n)\left(\mathbb{A}_{F}\right)$ with a minimal embedding

$$
{ }^{L} G \hookrightarrow G L_{N}(\mathbb{C}) \times W_{F}^{\prime},
$$

and let $\Pi$ be its transfer as an automorphic representation of $G L_{N}\left(\mathbb{A}_{F}\right)$. Then $\Pi$ is unitary isobaric.

It is then quite reasonable to conjecture that (cf. [21]):

CONJECTURe 5.7 ([21]). With notation as in Theorem 5.6, let $\pi$ be an arbitrary (unitary) cuspidal representation of a classical group. Let $\Pi$ be its conjectural functorial transfer with respect to a minimal embedding as before. Then $\pi$ is tempered if and only if $\Pi$ is unitary isobaric.

Observe that we are carefully distinguishing between "unitary isobaric" and "tempered isobaric" and in fact, if we take $\mathbf{G}=G L_{N}$ and $i$ equal to the identity map, then our conjecture is equivalent to the standard Ramanujan conjecture. 
We shall now explain the recent progress towards these conjectures for classical groups. Our main reference will be Section 10 of [21] as well as Section 8 of [48].

Let $\Pi=\otimes_{v} \Pi_{v}$ be a unitary cuspidal representation of $G L_{m}\left(\mathbb{A}_{F}\right)$. If $v$ is a place of $F$, then $\Pi_{v}$ is a unitary generic representation of $G L_{m}\left(F_{v}\right)$ and consequently can be written as

$$
\Pi_{v} \cong \operatorname{Ind}\left(\Pi_{1, v}|\operatorname{det}|^{a_{1}, v} \otimes \ldots \otimes \Pi_{t, v}|\operatorname{det}|^{a_{t, v}}\right)
$$

with $a_{1, v}>\ldots>a_{t, v}$ and each $\Pi_{i, v}$ tempered. We will say $\Pi$ satisfies condition $H\left(\theta_{m}\right)$ with $\theta_{m} \geq 0$ if for all places $v$ the exponents in $\Pi_{v}$ satisfy

$$
-\theta_{m} \leq a_{i, v} \leq \theta_{m} .
$$

As mentioned earlier the following result is due to Luo, Rudnick and Sarnak:

TheOREm 5.8 ([69]). Cuspidal representations of $G L_{m}\left(\mathbb{A}_{F}\right)$ satisfy $H\left(\frac{1}{2}-\frac{1}{m^{2}+1}\right)$.

Now let $\pi=\otimes \pi_{v}$ be a globally generic cuspidal representation of $G=\mathbf{G}\left(\mathbb{A}_{F}\right)$, where $\mathbf{G}$ is either a split classical group or $\mathbf{G}=U(n, n)$ for some natural number $n$. Then (cf. $[54,72,100])$

$$
\pi_{v} \simeq \operatorname{Ind}\left(\tau_{1, v}|\operatorname{det}|^{b_{1, v}} \otimes \ldots \otimes \tau_{t, v}|\operatorname{det}|^{b_{t, v}} \otimes \tau_{0, v}\right),
$$

where each $\tau_{i, v}$ is a tempered representation of an appropriate $G L$-group and $\tau_{0, v}$ is one of smaller rank but of same type classical group. We then say that $\pi$ satisfies $H(\theta(\mathbf{G})), \theta(\mathbf{G}) \geq 0$ a real number, if

$$
-\theta(\mathbf{G}) \leq b_{i, v} \leq \theta(\mathbf{G})
$$

The following is the content of Corollaries 10.1 and 10.2 of [21] and Theorem 8.1 of [48].

THEOREM $5.9([21,48])$. Let $\pi$ be a globally generic cuspidal representation of $G=\mathbf{G}\left(\mathbb{A}_{F}\right)$, where $\mathbf{G}$ is either a split classical group or $U(n, n)$. Let $N$ correspond to the minimal embedding of $L$-groups as before. Then $\pi$ satisfies condition $H\left(\frac{1}{2}-\frac{1}{N^{2}+1}\right)$. In particular, if the Ramanujan conjecture holds for general linear groups over a number field, then it holds for all the globally generic cuspidal representations of $G$.

REMARK 5.10. It is clear that as soon as one proves the analogue of Theorem 5.6 for other groups whose L-groups still have a classical derived group (cf. paragraph 4.4), one can fairly quickly conclude an analogue of Theorem 5.9 for them. Estimates obtained from the bound $\frac{1}{2}-\frac{1}{N^{2}+1}$ for $G L_{N}\left(\mathbb{A}_{F}\right)$ for these groups which are consequences of the work of Luo, Rudnick and Sarnak ([69], see Theorem 5.8 here) and the cases of functoriality discussed in Section 4, are quite deep and beat earlier bounds for these groups by far. Moreover, they provide uniform bounds below 1/2 for generic cusp forms on these groups that should have useful applications.

REMARK 5.11. When $F$ is a function field, Theorem 5.8 is valid maximally, i.e., $H(0)$ holds for all the cusp forms on $G L_{N}\left(\mathbb{A}_{F}\right)$. This is due to Lafforgue [58, 59] as explained earlier. Consequently, when the cases of functoriality discussed in Section 5 are established for $G=\mathbf{G}\left(\mathbb{A}_{F}\right)$, where $F$ is a function field, then similar arguments can be applied to establish the Ramanujan conjecture for all such groups. 
6. The Method. There are several methods for establishing functoriality. None is capable of proving it in general in its present form. The method used in proving the cases of functoriality discussed in Section 4 is that of $L$-functions. More precisely, one applies certain converse theorems of Cogdell and Piatetski-Shapiro [22, 24] to analytic properties of automorphic $L$-functions established through Langlands-Shahidi method $[32,45,82,83,84,85,86,87]$.

Converse theorems are very powerful when the target group is $G L_{N}$, i.e., when one attempts to prove the functoriality for a $L$-homomorphism as in (3.1). We shall now explain one of its versions $[22,24]$. We refer to $[18,19]$ for excellent expositions of the theory.

Let $S$ be a finite set of finite places of $F$. Fix a natural number $m$. Let $\mathcal{A}_{0}(m)$ denote the set of all the cuspidal representations of $G L_{m}\left(\mathbb{A}_{F}\right)$. Let

$$
\mathcal{A}_{0}^{S}(m)=\left\{\tau=\otimes_{v} \tau_{v} \mid \tau \in \mathcal{A}_{0}(m) \text { and } \tau_{v} \text { is unramified for } v \in S\right\} .
$$

Denote by $\Pi=\otimes_{v} \Pi_{v}$ an irreducible admissible representation of $G L_{N}\left(\mathbb{A}_{F}\right)$, where $N$ is a natural number. If $\omega_{\Pi_{v}}$ denotes the central character of $\Pi_{v}$, we will assume:

$$
\omega_{\Pi}=\otimes_{v} \omega_{\Pi_{v}} \text { is a grössencharacter, }
$$

i.e., a continuous character of $F^{*} \backslash \mathbb{A}_{F}^{*}$.

There is a local theory of Rankin product $L$-functions [40, 42, 43, 83] (cf. [18, 19] for excellent surveys) which attaches to each pair $\left(\tau_{v}, \Pi_{v}\right)$ of irreducible admissible representations of $G L_{m}\left(\mathbb{A}_{F}\right) \times G L_{N}\left(\mathbb{A}_{F}\right)$, a local $L$-function $L\left(s, \tau_{v} \times \Pi_{v}\right)$ and a local root numbers $\varepsilon\left(s, \tau_{v} \times \Pi_{v}, \psi_{v}\right)$, where $\psi=\otimes_{v} \psi_{v}$ is a non-trivial character of $F \backslash \mathbb{A}_{F}$. In fact, in view of the recent remarkable results of Harris-Taylor [35] and Henniart [36], they are simply Artin factors. More precisely, if

$$
\theta_{v}: W_{F_{v}}^{\prime} \longrightarrow G L_{m}(\mathbb{C})
$$

and

$$
\Theta_{v}: W_{F_{v}}^{\prime} \longrightarrow G L_{N}(\mathbb{C})
$$

are respectively the $m$-dimensional and the $N$-dimensional representations of $W_{F_{v}}^{\prime}$ attached to $\tau_{v}$ and $\Pi_{v}$ as discussed in Section 3, then

$$
L\left(s, \pi_{v} \times \Pi_{v}\right)=L\left(s, \theta_{v} \otimes \Theta_{v}\right)
$$

and

$$
\varepsilon\left(s, \pi_{v} \times \Pi_{v}, \psi_{v}\right)=\varepsilon\left(s, \theta_{v} \otimes \Theta_{v}, \psi_{v}\right) .
$$

Here the $L$-functions and root numbers in the right hand sides of (6.0.5) and (6.0.6) are those of Artin attached to the $m N$-dimensional representation $\theta_{v} \otimes \Theta_{v}$ of $W_{F_{v}}^{\prime}$ (cf. $[60,65,85,99])$.

We now consider

$$
L(s, \tau \times \Pi)=\prod_{v} L\left(s, \tau_{v} \times \Pi_{v}\right)
$$

and

$$
\varepsilon(s, \tau \times \Pi)=\prod_{v} \varepsilon\left(s, \tau_{v} \times \Pi_{v}, \psi_{v}\right)
$$


While (6.0.8) is a finite product as $\varepsilon\left(s, \tau_{v} \times \Pi_{v}, \psi_{v}\right)=1$ for almost all $v$, for (6.0.7) to make any sense we need to assume

$$
\text { The infinite product } \prod_{v} L\left(s, \tau_{v} \times \Pi_{v}\right) \text { converges for Re(s) sufficiently large. }
$$

We shall now state a very powerful version of converse theorems proved in [22, 24] (cf. $[18,19]$ for surveys).

Theorem 6.1 (Converse Theorem $[22,24]$ ). 1) Let $\Pi=\otimes \Pi_{v}$ be an irreducible admissible representation of $G L_{N}\left(\mathbb{A}_{F}\right)$ satisfying (6.0.2) and (6.0.9). Suppose that for all $\tau \in \mathcal{A}_{0}^{S}(m) \otimes \eta$, where $\eta$ is a grössencharacter of $\mathbb{A}_{F}^{*}$ and $1 \leq m \leq$ $N-1, L(s, \tau \times \Pi)$ is nice, i.e., it satisfies

a) $L(s, \tau \times \Pi)$ and $L(s, \tilde{\tau} \times \tilde{\Pi})$ extend to entire functions of $s \in \mathbb{C}$,

b) $L(s, \tau \times \Pi)$ and $L(s, \tilde{\tau} \times \tilde{\Pi})$ are bounded in vertical strips of finite width, and

c) $L(s, \tau \times \Pi)$ satisfies the functional equation

$$
L(s, \tau \times \Pi)=\varepsilon(s, \tau \times \Pi) L(1-s, \tilde{\tau} \times \tilde{\Pi}),
$$

where $\tilde{\tau}$ and $\tilde{\Pi}$ are contragredients of $\tau$ and $\Pi$, respectively. Then there exists an automorphic representation $\Pi^{\prime}=\otimes_{v} \Pi_{v}^{\prime}$ of $G L_{N}\left(\mathbb{A}_{F}\right)$ such that $\Pi_{v} \simeq \Pi_{v}^{\prime}$ for all $v \notin S$.

2) A similar statement is true even if we restrict ourselves to $1 \leq m \leq N-2$.

To use Theorem 6.1 to establish functoriality, one needs to construct a candidate for $\Pi$ which satisfies (6.0.2), (6.0.9) and conditions a), b) and c) of Theorem 6.1. In what follows we shall sketch how the functoriality for split groups for which ${ }^{L} G_{D}^{0}$ are classical are proved (cf. paragraphs 4.3 and 4.4). Although there are a number of well-written expository articles on how the cases of functoriality in paragraphs 4.1 and 4.2 are proved [4, 18, 31, 37, 76, 91, 92, 94], we will also briefly discuss them at the end due to their significance.

Let $\pi=\otimes_{v} \pi_{v}$ be a globally generic cuspidal representation of $G=\mathbf{G}\left(\mathbb{A}_{F}\right)$, where $\mathbf{G}$ is a split connected reductive group for which ${ }^{L} G_{D}^{0}$, the derived group of the connected component of its $L$-group, is a classical group. Our aim is to establish functoriality for the natural embeddings (4.3.1) and (4.4.1).

For almost all the places $v$ of $F$ including all the archimedean ones, the parametrization problem is solved [57, 65, 66] (Remark 3.2). With notation as in Section 3, we then choose $\Pi_{v}$ to be the representation of $G L_{N}\left(F_{v}\right)$ attached to $i \cdot \theta_{v}$, where

$$
\theta_{v}: W_{F_{v}}^{\prime} \longrightarrow{ }^{L} G
$$

is the parameter for $\pi_{v}$. Notice that for the cases of paragraph $4.4, \Pi_{v}$ then satisfies

$$
\Pi_{v} \simeq \tilde{\Pi}_{v} \otimes \omega_{\pi_{v}}
$$

At all other places we take $\Pi_{v}$ to be any irreducible admissible representation, except for its central character, which we require to satisfy

$$
\omega_{\Pi_{v}}=\omega_{\pi_{v}}^{n} .
$$

Here $n$ is the semisimple rank of $\mathbf{G}$. We refer to [7] for more details. When $\mathbf{G}$ is itself a classical group we will require $\omega_{\Pi_{v}}=1$. 
REMARK 6.2. Remarks are in order. First is that (6.0.11) implies that $\omega_{\Pi_{v}} / \omega_{\pi_{v}}^{n}$ is in fact a quadratic character $\mu_{v}$ and (6.0.12) corresponds to the case that $\mu_{v} \equiv 1$ for all $v$. Next, we should mention that such transfers are aimed at characterizing automorphic representations $\Pi=\otimes_{v} \Pi_{v}$ of $G L_{N}\left(\mathbb{A}_{F}\right)$ which satisfy

$$
\Pi \simeq \tilde{\Pi} \otimes \omega
$$

for some grössencharacter $\omega$. In fact, the theory of twisted endoscopy as developed by Kottwitz, Langlands and Shelstad [55, 67] sets the foundation for approaching this problem by means of Arthur's (twisted) trace formula [3]. When $\omega \equiv 1$, one will be dealing with the standard twisted endoscopy and then the self dual automorphic forms of $G L_{N}\left(\mathbb{A}_{F}\right)$ are expected to come from different classical groups. Those $\Pi$ for which $\omega_{\Pi}=1$ should be functorial transfers from split classical groups, while if $\omega_{\Pi}$ is a non-trivial (quadratic) character, then $\Pi$ should come from a form on $S O_{N}^{*}\left(\mathbb{A}_{F}\right)$, where $S O_{N}^{*}$ is the quasisplit form defined by the field extension attached to $\omega_{\Pi}$. This automatically requires $N$ to be even.

For $\omega \neq 1$, it is easily seen that if $N$ is even, then $\omega_{\Pi} / \omega^{N / 2}=\mu$ is a quadratic character. This time one expects $\Pi$ to be a transfer from a form on a GSpin ${ }_{m}^{*}\left(\mathbb{A}_{F}\right)$, where $N=2[\mathrm{~m} / 2]$. The quasisplit forms GSpin ${ }_{m}^{*}$ correspond to quadratic extensions defined by different $\mu$ 's and $N=m$; in particular $m$ is even. The cases we are addressing here correspond to $\mu \equiv 1$. We refer to [7] for details.

On the other hand if $N=2 n+1$ is odd, then it is easily checked that $\omega$ is square of a grössencharacter $\eta$ and therefore $\Pi \otimes \eta^{-1} \simeq\left(\Pi \otimes \eta^{-1}\right)^{\sim}$. We are therefore back in the case of standard twisted endoscopy and $\Pi \otimes \eta^{-1}$ should be a transfer from a classical group ( $S p_{2 n}$ to be precise).

We finally point out that if $N=2 n+1$ and $\Pi$ is self-dual with a (quadratic) central character $\omega_{\Pi}$ which is not trivial, then $\Pi \otimes \omega_{\Pi}$ is a self-dual representation which has a trivial central character. We therefore conclude that if $N$ is odd, $N=2 n+1$, then all the automorphic representations of $G L_{N}\left(\mathbb{A}_{F}\right)$ which satisfy (6.0.13) are twists of self-dual representations with trivial central characters and therefore twists of those which are transfers of forms on $S p_{2 n}\left(\mathbb{A}_{F}\right)$. For those which are transfers from globally generic forms on $S p_{2 n}\left(\mathbb{A}_{F}\right)$, this transfer was established in [21].

Having fixed $\Pi=\otimes_{v} \Pi_{v}$ we can clearly define $L(s, \tau \times \Pi)$ and $\varepsilon(s, \tau \times \Pi)$ by means of (6.0.7) and (6.0.8) for every $\tau \in \mathcal{A}_{0}^{S}(m) \otimes \eta$, where $1 \leq m \leq N-1$ and $\eta$ is a grössencharacter. To apply the converse theorem we need to show that $L(s, \tau \times \Pi)$ and $\varepsilon(s, \tau \times \Pi)$ are nice, i.e., satisfy the conditions of Theorem 6.1 for some $S$ and $\eta$.

The $L$-functions $L(s, \tau \times \Pi)$ are defined by means of an infinite product of local Artin $L$-functions and á priori nothing intelligent can be said about them. But the remarkable fact is that they are in fact equal to another family of $L$-functions defined by the Langlands-Shahidi method. It is these $L$-functions that satisfy conditions of Theorem 6.1 and to which we can apply the theorem to establish functoriality.

To explain, we now go back to our original representation $\pi=\otimes_{v} \pi_{v}$. We recall that $\pi$ is a globally generic cuspidal representation of $G=\mathbf{G}\left(\mathbb{A}_{F}\right)$.

We first observe that for every finite place $v$ for which $\pi_{v}, \tau_{v}, \psi_{v}$ and $\eta_{v}, \eta=\otimes_{v} \eta_{v}$, are all unramified,

$$
L\left(s, \tau_{v} \times \pi_{v}\right)=L\left(s, \tau_{v} \times \Pi_{v}\right)
$$

and

$$
\varepsilon\left(s, \tau_{v} \times \pi_{v}, \psi_{v}\right)=\varepsilon\left(s, \tau_{v} \times \Pi_{v}, \psi_{v}\right)=1,
$$


for each $\tau \in \mathcal{A}_{0}(m) \otimes \eta$. For the archimedean places (6.0.14) and the first equality in (6.0.15) are special cases of the results proved in [85]. They are valid for the irreducible admissible generic representations $\tau_{v}, \pi_{v}$ and $\Pi_{v}$, where $\Pi_{v}$ is the local transfer of $\pi_{v}$ parametrized by $i \cdot \theta_{v}$, where $\theta_{v}$ is as in (6.0.10) (cf. [65]). We point out that $\Pi_{v}$ need not necessarily be generic.

Let $n$ be the semisimple rank of $\mathbf{G}$. We will write $\mathbf{G}_{n}=\mathbf{G}$. In each of the present cases, there exists a connected (split) reductive group $\mathbf{H}$ of the same type as $\mathbf{G}$, but of semisimple rank $m+n$ which contains $\mathbf{M}=G L_{m} \times \mathbf{G}_{n}$ as a Levi factor. One then considers $\tilde{\tau} \otimes \pi$, with $\tau \in \mathcal{A}_{0}^{S}(m) \otimes \eta$ as a generic cuspidal representation of $M=\mathbf{M}\left(\mathbb{A}_{F}\right)$.

Fixing an appropriate Borel subgroup $\mathbf{B}_{\mathbf{H}}$ defined over $F$ in $\mathbf{H}$, one can then consider a standard parabolic subgroup $\mathbf{P} \supset \mathbf{B}_{\mathbf{H}}$ which has $\mathbf{M}$ as a Levi factor. The parabolic $\mathbf{P}$ being a maximal, one then attaches a "rank one" Eisenstein series $E_{s}(h)$ to $(\mathbf{H}, \mathbf{M}, \tilde{\tau} \otimes \pi)$ by means of Langlands theory of Eisenstein series [1, 10, 34, 62, $63,71,73]$, where the complex number $s$ is identified as the corresponding complex parameter.

Given a finite set of places $S_{0}$, where everything is unramified for $v \notin S_{0}$, we let

$$
L^{S_{0}}(s, \tau \times \pi)=\prod_{v \notin S_{0}} L\left(s, \tau_{v} \times \pi_{v}\right) .
$$

Normalizing the identification of $s$ as the complex parameter of $E_{s}$, it follows from the theory of constant terms [34, 62, 63, 71, 86, 87] that the constant term of $E_{s}$ along $\mathbf{P}$, i.e.,

$$
\int_{\mathbf{U}_{\mathbf{P}}(F) \backslash \mathbf{U}_{\mathbf{P}}\left(\mathbb{A}_{F}\right)} E_{s}(u h) d u
$$

has the ratio

$$
\mathcal{A}_{S_{0}}(s, \tau, \pi) L^{S_{0}}(s, \tau \times \pi) L^{S_{0}}\left(2 s, \tau, r_{2}\right) / L^{S_{0}}(s+1, \tau \times \pi) L^{S_{0}}\left(2 s+1, \tau, r_{2}\right)
$$

as its main term. Here $\mathbf{U}_{\mathbf{P}}$ is the unipotent radical of $\mathbf{P}$ and $\mathcal{A}_{S_{0}}(s, \tau, \pi)$ is a product of local intertwining operators over all $v \in S_{0}$. Moreover, $L^{S_{0}}\left(\cdot, \tau, r_{2}\right)$ is another infinite product attached to $\tau$ and a representation $r_{2}$ of the $L$-group $G L_{m}(\mathbb{C})$ of $G L_{m}$ which is a twist of either $\Lambda^{2}$ or $\operatorname{Sym}^{2}$ representations of $G L_{m}(\mathbb{C})$ depending on the case. The $L$-function $L^{S_{0}}\left(s, \tau, r_{2}\right)$ appears as the only $L$-function present in the constant term of the Eisenstein series induced from $\tau$ considered as a representation of the Siegel Levi (or its variant for GSpin groups) $G L_{m}$ inside a group $\mathbf{H}$ as above of semisimple rank $m$. Its analytic properties are then deduced from this setting.

Moreover, it can be shown that the non-constant term of $E_{s}(h)$ with respect to a "generic" character $\tilde{\chi}$ of the adelic points $\tilde{U}$ of the unipotent radical $\tilde{\mathbf{U}}$ of $\mathbf{B}_{\mathbf{H}}$ for which $\tilde{\chi} \mid \tilde{U} \cap G_{n}=\chi$, where $\chi$ is as in Section 2, is given, up to a product of local "Whittaker functions" by

$$
L^{S_{0}}\left(2 s+1, \tau, r_{2}\right)^{-1} L^{S_{0}}(s+1, \tau \times \pi)^{-1}
$$

(cf. $[16,84,86,92])$.

The thrust of the Langlands-Shahidi method is to exploit the analytic properties of Eisenstein series and its constant and non-constant terms to deduce the analytic properties of these $L$-functions and in particular those needed in Theorem 6.1. This 
is done in the method in the generality of all the $L$-functions that appear in constant terms of Eisenstein series attached to generic representations of Levi factors of any quasisplit group and is therefore quite general. But here we will continue with our special cases discussed in 4.3 and 4.4 .

To this end, we restrict the definitions of local $L$-functions and root numbers given in [87] to our cases and note that one can define $L\left(s, \tau_{v} \times \pi_{v}\right)$ and $\varepsilon\left(s, \tau_{v} \times \pi_{v}, \psi_{v}\right)$ in such a way that

$$
L(s, \tau \times \pi)=\varepsilon(s, \tau \times \pi) L(1-s, \tilde{\tau} \times \tilde{\pi}),
$$

where

$$
L(s, \tau \times \pi)=\prod_{v} L\left(s, \tau_{v} \times \pi_{v}\right)
$$

and

$$
\varepsilon(s, \tau \times \pi)=\prod_{v} \varepsilon\left(s, \tau_{v} \times \pi_{v}, \psi_{v}\right) .
$$

The definition of these local factors is quite delicate and requires induction and local-global methods [86, 87]. Moreover, when $v$ is archimedean, it is shown in [85] that the factors are those of Artin attached through parametrization [65] as discussed in Remark 3.2.

Condition (6.0.2) is now a consequence of the fact $\omega_{\Pi}=\omega_{\pi}^{n}$ and is therefore a grossencharacter ( $\omega_{\Pi} \equiv 1$ in the cases of split classical groups.) Condition (6.0.9) follows from the absolute convergence of $L^{S_{0}}(s, \tau \times \pi)$ for $\operatorname{Re}(s)>>0$.

To prove that condition a) of Theorem 6.1 is true, we choose $S$ and $\eta$ appropriately. In fact, we let $S$ be a non-empty set of finite places which includes all the places $v$ where $\pi_{v}$ or $\psi_{v}$ (this is not serious) are ramified and choose $\eta$ such that $\eta_{v}$ is sufficiently ramified for each $v \in S$. (For verifying a) one place is enough. But due to the issue of stability of root numbers which we have to deal with as we explain later, we need to have it at all the ramified places.) The representation $\tilde{\tau}_{v} \otimes \pi_{v}$ will not be equivalent to its conjugate under the non-trivial element of the Weyl group of the connected center (split component) of $\mathbf{M}$ in $\mathbf{H}$ if $\eta_{v}$ is sufficiently ramified, as one can verify that they will not even have equal central characters. It then follows from the theory of Eisenstein series that $E_{s}(h)$ is entire $[34,45,63,71]$ from which holomorphy of both $L$-functions follows (through induction). This was first observed by Kim in certain special cases in [45]. A general statement and proof for all the $L$-functions obtained from our method is given in [53], Proposition 2.1.

The boundedness in vertical strips for $L(s, \tau \times \pi)$ is a special case of the general Theorem 4.1 of [32]. Consequently both parts a) and b) are verified for $L(s, \tau \times \Pi)$ as well.

Condition c) of Theorem 6.1 is quite subtle and has been the biggest challenge so far. To conclude the functional equation (6.1.1) from (6.0.20), we need to know that

$$
L\left(s, \tau_{v} \times \pi_{v}\right)=L\left(s, \tau_{v} \times \Pi_{v}\right)
$$

and

$$
\varepsilon\left(s, \tau_{v} \times \pi_{v}, \psi_{v}\right)=\varepsilon\left(s, \tau_{v} \times \Pi_{v}, \psi_{v}\right)
$$

for all $v \in S_{0}$. But this is a very difficult claim to verify. The local factors on the right are those of Artin, i.e., those attached to Weil-Deligne group (as in [99]) 
through the local Langlands correspondence [35, 36, 65] discussed in Remark 3.2, and are well understood, while the ones on the left are those defined by the LanglandsShahidi method, and although are well-suited to parametrization and satisfy the delicate multiplicativity properties $[87,88,92]$ expected from them, they are defined through complicated harmonic analytic methods and different inductions [86, 87, 92]. Consequently, although they are eventually proved to be equal, á priori have nothing to do with each other!

If $v$ is in $S_{0}$ but not $S$, then all $\pi_{v}$ 's are unramified and the equalities follow from multiplicativity of both factors on both sides of (6.0.23) and (6.0.24) under parabolic induction, since $\pi_{v}$ 's are subrepresentations of principal series (cf. [87, 88]).

It is for $v \in S$ were the issue of twisting by a highly ramified $\eta_{v}$ is necessary. The equality (6.0.23) of $L$-functions for $\eta_{v}$ highly ramified becomes a special case of the main result of [90], since then both $L$-functions become identically equal to 1 . This is the local version of Proposition 2.1 of [53] and is proved using the same techniques.

It is the equality (6.0.24) for a highly ramified $\eta_{v}$ which is complicated and subtle, but there is no way around it and finally saves the day. What is proved is that either side of (6.0.24) depends only on the corresponding central characters if $\eta_{v}$ is highly ramified. It then becomes an easy exercise to compute their "stable" forms by assuming $\pi_{v}$ to be a principal series to which then one can apply multiplicativity. They become equal!

To prove that either side depends only on central characters, we proceed as follows. Since $\tau_{v}$ is unramified, one can apply "multiplicativity" to either side of (6.0.24) to reduce to the case where $\mathbf{M}=G L_{1} \times \mathbf{G}_{n}$. The problem then becomes to show the stability in this case. This is very delicate. The first case of this was proved by Cogdell and Piatetski-Shapiro for the case of $G L_{1} \times S O(2 n+1)$ in [23]. There they expressed the root number $\varepsilon\left(s, \eta_{v} \times \pi_{v}, \psi_{v}\right)$ or rather the $\gamma$-function $\gamma\left(s, \eta_{v} \times \pi_{v}, \psi_{v}\right)$ defined by

$$
\gamma\left(s, \eta_{v} \times \pi_{v}, \psi_{v}\right)=\varepsilon\left(s, \eta_{v} \times \pi_{v}, \psi_{v}\right) L\left(1-s, \eta_{v}^{-1} \times \tilde{\pi}_{v}\right) / L\left(s, \eta_{v} \times \pi_{v}\right)
$$

as the Mellin transform of an appropriate Bessel function attached to $\pi_{v}$ against the "Mellin variable" $\eta_{v}$. Using asymptotics of Bessel functions which they established in [23], they then showed the independence of $\gamma\left(s, \eta_{v} \times \pi_{v}, \psi_{v}\right)$ from $\pi_{v}$ (central character of $\pi_{v}$ is trivial, $S O(2 n+1)$ being adjoint), if $\eta_{v}$ is highly ramified. It should be pointed out that $\gamma\left(s, \eta_{v} \times \pi_{v}, \psi_{v}\right)$ in [23] was defined by means of an integral representation via Rankin-Selberg method.

While the theory of Bessel functions developed in [23] seems to be valid in the generality of general quasisplit groups, there are no nice expressions for $\gamma\left(s, \eta_{v} \times \pi_{v}, \psi_{v}\right)$ in any of other cases discussed in 4.3 and 4.4 coming from the Rankin-Selberg method. On the other hand as it was shown in [93], there is a fairly general expression for $\gamma$-factors $\gamma\left(s, \eta_{v} \times \pi_{v}, \psi_{v}\right)$ or rather their building blocks, the corresponding "local coefficients" (cf. Theorem 3.5 of [87]), which expresses them again as the Mellin transform of the corresponding Bessel functions verses the Mellin variable $\eta_{v}$, when $\eta_{v}$ is sufficiently ramified (Theorem 6.2 of [93]).

It is by means of Theorem 6.2 of [93] which one proves the stability of $\gamma\left(s, \eta_{v} \times\right.$ $\left.\pi_{v}, \psi_{v}\right)$ and consequently $\varepsilon\left(s, \eta_{v} \times \pi_{v}, \psi_{v}\right)$ for highly ramified $\eta_{v}$ in [21, 48] and [7] for the cases discussed in 4.3 and 4.4, respectively. In each case in [7, 21, 48], a lot more work is needed to get the expression in [93] to look like a genuine Mellin transform. In particular, in [7] one needs to do quite a lot of calculations using abstract theory of roots since no convenient matrix presentations are available for spinor groups.

Having established (6.0.23) and (6.0.24) for every $v$, functional equation (6.0.20) then implies (6.1.1). We can now apply Theorem 6.1 to conclude the existence of 
$\Pi^{\prime}=\otimes_{v} \Pi_{v}^{\prime}$ for which $\Pi_{v}^{\prime} \simeq \Pi_{v}$ for all $v \notin S$, thus establishing Conjecture 3.1 for embeddings (4.3.1) and (4.4.1) in 4.3 and 4.4.

We shall now very briefly explain how the automorphy of $\pi_{1} \otimes \pi_{2}$ is proved, where $\pi_{1}=\otimes_{v} \pi_{1 v}$ and $\pi_{2}=\otimes_{v} \pi_{2 v}$ are cuspidal representations of $G L_{2}\left(\mathbb{A}_{F}\right)$ and $G L_{3}\left(\mathbb{A}_{F}\right)$, respectively, discussed in 4.1 . We refer to $[31,37,46,51,53,76,91,94]$ for detailed proofs and other expository articles on this problem.

The converse theorem to use is Theorem 6.1.b and in fact we need to show

$$
\begin{aligned}
L\left(s, \tau \times\left(\pi_{1} \otimes \pi_{2}\right)\right) & =\prod_{v} L\left(s, \tau_{v} \times\left(\pi_{1 v} \otimes \pi_{2 v}\right)\right) \\
& =\prod_{v} L\left(s, \rho_{v} \otimes \theta_{1 v} \otimes \theta_{2 v}\right)
\end{aligned}
$$

is nice for all $\tau=\otimes_{v} \tau_{v} \in \mathcal{A}_{0}^{S}(m) \otimes \eta, 1 \leq m \leq 4$, and an appropriate grössencharacter $\eta$. Here $\theta_{i v}: W_{F_{v}}^{\prime} \longrightarrow G L_{i+1}(\mathbb{C}), i=1,2$, are complex representations parametrizing $\pi_{i v}$ and $\rho_{v}: W_{F_{v}}^{\prime} \longrightarrow G L_{m}(\mathbb{C})$ is that for $\tau_{v}$ as discussed in 4.1. The set $S$ consists of all those finite places $v$ where either $\pi_{1 v}$ or $\pi_{2 v}$ is ramified.

On the other hand, using the Langlands-Shahidi method discussed earlier, we can choose a triple $(\mathbf{G}, \mathbf{M}, \pi)$ of a reductive group $\mathbf{G}$ with a Levi subgroup $\mathbf{M}$ together with a cuspidal representation $\pi$ of $M=\mathbf{M}\left(\mathbb{A}_{F}\right)$ such that the "main" $L$-function appearing in the constant term of the corresponding Eisenstein series has $L\left(s, \tau_{v} \times\right.$ $\left.\left(\pi_{1 v} \otimes \pi_{2 v}\right)\right)$ as its local factor at least when $v=\infty$ or when all the data are unramified. To be explicit, one takes $\mathbf{G}$ to be $G L_{5}, \operatorname{Spin}_{10}, E_{6}^{s c}$ and $E_{7}^{s c}$ according as $m=1,2,3,4$, respectively. The Levi subgroup $\mathbf{M}$ must then be chosen so that its derived group be respectively equal to $S L_{3} \times S L_{2}, S L_{3} \times S L_{2} \times S L_{2}, S L_{3} \times S L_{2} \times S L_{3}$ and $S L_{3} \times$ $S L_{2} \times S L_{4}$. In each case there is an injection from $M=\mathbf{M}\left(\mathbb{A}_{F}\right)$ into $G L_{3}\left(\mathbb{A}_{F}\right) \times$ $G L_{2}\left(\mathbb{A}_{F}\right) \times G L_{m}\left(\mathbb{A}_{F}\right), 1 \leq m \leq 4$. The representation $\pi$ is then simply a constituent of the discrete sum $\pi_{1} \otimes \pi_{2} \otimes \tau \mid M$ whose choice is irrelevant. The symbol "sc" signifies the simply connected form of the given group. They are all assumed to be split over $F$.

Again the method allows us to define local $L$-functions and root numbers everywhere and leads to a proof of necessary properties of the completed $L$-functions. Theorem 6.1.b then applies, proving the existence of an automorphic representation $\Pi^{\prime}=\otimes_{v} \Pi_{v}^{\prime}$ such that $\Pi_{v}^{\prime}=\pi_{1 v} \otimes \pi_{2 v}$ at almost all the places, including the archimedean ones.

It takes quite a bit of effort to show that $\Pi_{v}^{\prime}=\pi_{1 v} \otimes \pi_{2 v}$ at every other place, including the use of both normal and non-normal base change [5, 41], as well as even some local theory of types [15]. The local $L$-functions and root numbers at the ramified places defined from the Langlands-Shahidi method then become equal to the Artin factors attached to $\rho_{v} \otimes \theta_{1 v} \otimes \theta_{2 v}$ at all the places.

\section{REFERENCES}

[1] J. ARthur, Eisenstein series and trace formula, in Automorphic Forms, Representations and $L$-functions, Proc. Sympos. Pure Math., 33, Part 1 (1979), pp. 253-274.

[2] J. ARThur, On some problems suggested by the trace formula, in Lie Group Representations II, Proceedings, Univ. of Maryland 1982-1983, B. Herb, S. Kudla, R. Lipsman and J. Rosenberg eds., Springer LN 1041, pp. 1-49.

[3] J. ARThUR, Unipotent automorphic representations: Global motivation, in Automorphic Forms, Shimura Varieties and $L$-functions, Vol. I, Editors L. Clozel and S.J. Milne, Perspect. Math. 10, Academic Press, Boston, 1990, pp. 1-75. 
[4] J. Arthur, The principle of functoriality, Bull. Amer. Math. Soc. (N.S.), 40:1 (2002), pp. 39-53; Mathematical Challenges of the 21st century (Los Angeles, CA, 2000).

[5] J. Arthur And L. Clozel, Simple Algebras, Base Change, and the Advanced Theory of the Trace Formula, Ann. of Math. Studies 120, Princeton Univ. Press, Princeton, NJ, 1989.

[6] M. Asgari, Local L-functions for split spinor groups, Canad. J. Math., 54 (2002), pp. 673693.

[7] M. Asgari And F. Shahidi, Generic transfer for general spin groups, preprint.

[8] D. Blasius, Hilbert modular forms and the Ramanujan conjecture, preprint.

[9] A. Borel, Automorphic L-functions, Proc. Sympos. Pure Math., 33, Part 2 (1979), pp. 27-61.

[10] A. Bonel, Automorphic Forms on $S L_{2}(\mathbb{R})$, Cambridge Tracts in Mathematics, Vol. 130, Cambridge University Press, 1997.

[11] A. Borel and N. Wallach, Continuous Cohomology, Discrete Subgroups, and Representations of Reductive Groups, Annals of Math. Studies 94, Princeton Univ. Press, 1980.

[12] J-L. Brylinski and J-P. Labesse, Cohomologie d'intersection et fonctions L de certaines varietés de Shimura, Ann. Sci. École Norm Sup., 17 (1984), pp. 361-412.

[13] D. Bump, W. Duke, J. Hoffstein, And H. Iwaniec, An estimate for the Hecke eigenvalues of Maass forms, IMRN, 4 (1992), pp. 75-81.

[14] D. Bump, D. Ginzburg And J. Hoffstein, The Symmetric Cube, Invent. Math., 125 (1996), pp. $413-449$.

[15] C. J. Bushnell and G. Henniart, On certain dyadic representations (Appendix to [52], Ann. of Math., 155 (2002), pp. 883-893.

[16] W. Casselman and J.A. ShaliKa, The unramified principal series of p-adic groups II, The Whittaker function, Comp. Math., 41 (1980), pp. 207-231.

[17] L. ClOzel, Spectral theory of automorphic forms, IAS/Park City Lecture Notes, Park City, Utah, 2002.

[18] J.W. Cogdell, L-functions and Converse Theorems for $G L(n)$, IAS/Park City Lecture Notes, Park City, Utah, 2002.

[19] J.W. Cogdell and I.I. Piatetski-Shapiro, Converse theorems, functoriality, and applications to number theory, Proceedings of the International Congress of Mathematicians, Vol. II (Beijing, 2002), Higher Ed. Press, Beijing, 2002, pp. 119-128.

[20] J.W. Cogdell, H. Kim, I.I. Piatetski-Shapiro and F. Shahidi, On lifting from classical groups to $G L_{N}$, Publ. Math. Inst. Hautes Études Sci., 93 (2001), pp. 5-30.

[21] J.W. Cogdell, H. Kim, I.I. Piatetski-Shapino and F. Shahidi, Functoriality for the classical groups, Publ. Math. Inst. Hautes Études Sci., 99 (2004), pp. 163-233.

[22] J.W. Cogdell and I.I. Piatetski-Shapiro, Converse theorems for $G L_{n}$, Publ. Math. Inst. Hautes Études Sci., 79 (1994), pp. 157-214.

[23] J.W. Cogdell and I.I. Piatetski-Shapiro, Stability of Gamma Factors for $S O(2 n+1)$, Manuscripta Math., 95 (1998), pp. 437-461.

[24] J.W. Cogdell and I.I. Piatetski-Shapiro, Converse theorems for $G L_{n} I I$, J. Reine Angew. Math., 507 (1999), pp. 165-188.

[25] P. Deligne, La conjecture de Weil. I, Publ. Math. Inst. Hautes Études Sci., 43 (1974), pp. 273-307.

[26] V.G. Drinfeld, Langlands' conjecture for GL(2) over functional fields, Proceedings of ICM, Helsinki, pp. 565-574, (1978).

[27] V.G. Drinfeld, The proof of Petersson's conjecture for GL(2) over a global field of characteristic p, Functional Analysis and its Applications, 22 (1988), pp. 28-43.

[28] W. Duke And H. Iwaniec, Estimates for coefficients of L-functions, I, Automorphic forms and analytic number theory (Montreal, PQ, 1989), Univ. Montréal, Montréal, QC, 1990, pp. $43-47$.

[29] S. Gelbart, Automorphic forms on adéle groups, Princeton University Press, Princeton, NJ, 1975, Annals of Mathematics Studies, no. 83.

[30] S. Gelbart AND H. JACQUet, A relation between automorphic representations of $G L(2)$ and GL(3), Ann. Sci. École Norm. Sup., 11:4 (1978), pp. 471-542.

[31] S. Gelbart and S. Miller, Riemann's zeta function and beyond, Bulletin of AMS, 41:1 (2003), pp. 59-112.

[32] S. Gelbart AND F. Shahidi, Boundedness of automorphic L-functions in vertical strips, J. Amer. Math. Soc., 14:1 (2001), pp. 79-107.

[33] D. Ginzburg, S. Rallis, And D. Soudry, Generic automorphic forms on $S O(2 n+1)$ : functorial lift to $G L(2 n)$, endoscopy, and base change, IMRN, 2001:14 (2001), pp. 729-764.

[34] Harish-Chandra, Automorphic forms on semisimple Lie groups, Notes by J.G.M. Mars. Lecture Notes in Mathematics, no. 62, Springer-Verlag, Berlin, 1968.

[35] M. HARRIS AND R. TAYLOR, The geometry and cohomology of some simple Shimura varieties, 
Annals of Mathematics Studies, Vol. 151, Princeton University Press, Princeton, NJ, 2001.

[36] G. Henniart, Une preuve simple des conjectures de Langlands pour $G L(n)$ sur un corps p-adique, Invent. Math., 139 (2000), pp. 439-455.

[37] G. Henniart, Progrés récents en fonctorialité de Langlands, Seminaire Bourbaki, Vol. 2000/2001, Astérisque No. 282 (2002), Exp. 890, pp. 301-322.

[38] R. Howe and I.I. Piatetski-Shapiro, A counter-example to the "generalized Ramanujan conjecture" for (quasi)-split groups, Proc. Sympos. Pure Math., 33, Part 1 (1979), pp. $315-322$.

[39] H. Iwaniec, Spectral methods of automorphic forms, 2nd ed., Graduate Studies in Mathematics, Vol. 53, American Mathematical Society, Providence, RI, 2002.

[40] H. Jacquet, I.I. Piatetski-Shapiro, and J.A. Shalika, Rankin-Selberg convolutions, Amer. J. Math., 105:2 (1983), pp. 367-464.

[41] H. Jacquet, I.I. Piatetski-Shapiro, and J.A. Shalika, Relévement cubique non normal, C.R. Acad. Sci. Paris Sér. I Math., 292 (1981), pp. 567-571.

[42] H. JaCQuet And J.A. Shalika, On Euler products and the classification of automorphic representations, I, Amer. J. Math., 103:3 (1981), pp. 499-558.

[43] H. JaCQuet And J.A. Shalika, On Euler products and the classification of automorphic representations, II, Amer. J. Math., 103 (1981), pp. 777-815.

[44] D. JiAng AND D. SoudRY, Generic representations and local Langlands reciprocity law for p-adic $S_{2 n+1}$, Contributions to Automorphic Forms, Geometry and Number Theory (Shalikafest 2002) (H. Hida, D. Ramankrishnan, and F. Shahidi, eds.), Johns Hopkins University Press, Baltimore, 2004, pp. 457-520.

[45] H. KIM, Langlands-Shahidi method and poles of automorphic L-functions: application to exterior square L-functions, Canad. J. Math., 51:4 (1999), pp. 835-849.

[46] H. KIM, Functoriality for the exterior square of $G L_{4}$ and the symmetric fourth of $G L_{2}$, with appendix 1 by D. Ramakrishnan and appendix 2 by H. Kim and P. Sarnak, J. Amer. Math. Soc., 16:1 (2002), pp. 139-183.

[47] H. KIM, On local L-functions and normalized intertwining operators, Canad. J. Math., to appear.

[48] H. Kim and M. Krishnamurthy, Base change lift from unitary groups to $G L_{N}$, preprint.

[49] H. Kim And P. SaRnak, Refined estimates towards the Ramanujan and Selberg conjectures, Appendix 2 to [46], J. Amer. Math. Soc., 16:1 (2002), pp. 175-181.

[50] H. Kim And F. Shahidi, Symmetric cube L-functions for $G L_{2}$ are entire, Ann. of Math., 150 (1999), pp. 645-662.

[51] H. Kim And F. Shahidi, Functorial products for $G L_{2} \times G L_{3}$ and functorial symmetric cube for $G L_{2}$, C.R. Acad. Sci. Paris Sér. I Math., 331:8 (2000), pp. 599-604.

[52] H. Kim AND F. Shahidi, Cuspidality of symmetric powers with applications, Duke Math. J., 112:1 (2002), pp. 177-197.

[53] H. KIm And F. Shahidi, Functorial products for $G L_{2} \times G L_{3}$ and the symmetric cube for $G L_{2}$, with an appendix by C.J. Bushnell and G. Henniart, Ann. of Math., 155:3 (2002), pp. 837-893.

[54] B. Kostant, On Whittaker vectors and representation theory, Invent. Math., 48 (1978), pp. $101-184$

[55] R. Kottwitz and D. Shelstad, Foundations of twisted endoscopy, Astérisque, 255 (1999), pp. 1-190.

[56] N. Kurokawa, Examples of eigenvalues of Hecke operators on Siegel cusp forms of degree two, Invent. Math., 49 (1978), pp. 149-165.

[57] J-P. Labesse, Cohomologie, L-groupes et fonctorialité, Comp. Math., 55 (1984), pp. 163-184.

[58] L. Lafforgue, Chtoucas de Drinfeld et correspondance de Langlands, Invent. Math., 147:1 (2002), pp. 1-241.

[59] L. Lafforgue, Chtoucas de Drinfeld, Formule des Traces d'Arthur-Selberg et Correspondence de Langlands, Proceedings of the International Congress of Mathematicians, Vol. I (Beijing, 2002), Higher Ed. Press, Beijing, 2002, pp. 383-400.

[60] R.P. Langlands, On Artin's L-functions, Rice Univ. Studies 56, Houston, TX, 1970.

[61] R.P. Langlands, Problems in the theory of automorphic forms, in Lecture Notes in Math. 170, Springer-Verlag, Berlin-Heidelberg-New York, 1970, pp. 18-86.

[62] R.P. Langlands, Euler products, Yale Mathematical Monographs, 1, Yale University Press, New Haven, CT, 1971.

[63] R.P. Langlands, On the functional equations satisfied by Eisenstein series, Lecture Notes in Mathematics, Vol. 544, Springer-Verlag, Berlin, 1976.

[64] R.P. Langlands, Automorphic representations, Shimura varieties, and motives. Ein 
Märchen, Proc. Sympos. Pure Math., 33, Part 2 (1979), pp. 205-246.

[65] R.P. LANGLANDS, On the classification of irreducible representations of real algebraic groups, Representation Theory and Harmonic Analysis on Semisimple Lie Groups, Math. Surveys Monogr., Vol. 31, Amer. Math. Soc., Providence, RI, 1989, pp. 101-170.

[66] R.P. Langlands, Representations of Abelian Algebraic Groups, Olga Taussky-Todd Memorial Issue, Pacific J. Math., (1997), pp. 231-250.

[67] R.P. Langlands and D. Shelstad, On the definition of transfer factors, Math. Ann., 278 (1987), pp. 219-271.

[68] W. Luo, Z. Rudnick, And P. SARnak, On Selberg's eigenvalue conjecture, Geom. Funct. Anal., 5:2 (1995), pp. 387-401.

[69] W. Luo, Z. RudNick ANd P. SARnAK, On the generalized Ramanujan conjecture for $G L(n)$, Automorphic forms, automorphic representations, and arithmetic, Proc. Sympos. Pure Math., 66 (1999), pp. 301-310.

[70] H. MAass, Über eine neue Art von nichtanalytischen automorphen Funktionen und die Bestimmung Dirichletscher Reihen durch Funktionalgleichungen, Math. Ann., 121 (1949), pp. $141-183$.

[71] C. Moeglin and J.-L. Waldspurger, Spectral decomposition and Eisenstein series, Cambridge Tracts in Mathematics, vol. 113, Cambridge University Press, Cambridge, 1995.

[72] G. MuIć, A proof of Casselman-Shahidi's conjecture for quasi-split classical groups, Canad. Math. Bull., 44 (2001), pp. 298-312.

[73] W. MüLLER, The trace class conjecture in the theory of automorphic forms, Ann. of Math., 130:3 (1989), pp. 473-529.

[74] I.I. Piatetski-Shapiro, Multiplicity one theorems, Proc. Sympos. Pure Math., 33, Part 1 (1979), pp. 209-212.

[75] D. Ramakrishnan, Modularity of the Rankin-Selberg L-series, and multiplicity one for $S L(2)$, Ann. of Math., 152 (2000), pp. 45-111.

[76] P. Sarnak, Spectra of hyperbolic surfaces, Bull. Amer. Math. Soc. (N.S.), 40 (2003), pp. 441-478.

[77] P. SARnaK, Notes on the generalized Ramanujan conjectures, Fields Institute Lectures, June 2003.

[78] I. Satake, Spherical functions and Ramanujan Conjecture, Proc. Sympos. Pure Math., 9 (1966), pp. 258-264.

[79] A. Selberg, On the estimation of Fourier coefficients of modular forms, Proc. Sympos. Pure Math., 8 (1965), pp. 1-15.

[80] J-P. SerRe, Abelian l-adic Representations and Elliptic Curves, W.A. Benjamin, New York, 1968.

[81] J-P. Serre, Appendix to [89], in Elliptic Curves and Related Topics, CRM Proc. Lecture Notes, Vol. 4, AMS, Providence, RI, 1994, pp. 175-180.

[82] F. ShAHIDI, Functional equations satisfied by certain L-functions, Comp. Math., 37 (1978), pp. 171-207.

[83] F. SHAHIDI, Fourier transforms of intertwining operators and Plancherel measures for GL $(n)$, Amer. J. Math., 106 (1984), pp. 67-111.

[84] F. SHAhidi, On certain L-functions, Amer. J. Math., 103:2 (1981), pp. 297-355.

[85] F. SHAhidi, Local coefficients as Artin factors for real groups, Duke Math. J., 52:4 (1985), pp. 973-1007.

[86] F. SHAHIDI, On the Ramanujan conjecture and finiteness of poles for certain L-functions, Ann. of Math., 127:3 (1988), pp. 547-584.

[87] F. Shahidi, A proof of Langlands' conjecture on Plancherel measures; complementary series for p-adic groups, Ann. of Math., 132:2 (1990), pp. 273-330.

[88] F. Shahidi, On multiplicativity of local factors, Festschrift in honor of I.I. Piatetski-Shapiro on the occasion of his sixtieth birthday, Part II (Ramat Aviv. 1989), Israel Math. Conf. Proc., 3, Weizmann, Jerusalem (1990), pp. 279-289.

[89] F. Shahidi, Symmetric power L-functions for $G L(2)$, Elliptic curves and related topics, with an appendix by J-P. Serre, CRM Proc. Lecture Notes, vol. 4, Amer. Math. Soc., Providence, RI, 1994, pp. 159-182.

[90] F. SHAhidI, Twists of a general class of L-functions by highly ramified characters, Canadian Math. Bull., 43 (2000), pp. 380-384.

[91] F. Shahid, Automorphic L-functions and functoriality, Proceedings of the International Congress of Mathematicians, Vol. II (Beijing, 2002), Higher Ed. Press, Beijing, 2002, pp. 655-666.

[92] F. Shahidi, Langlands-Shahidi Method, IAS/Park City Lecture Notes, Park City, Utah, 2002.

[93] F. ShAHIDI, Local coefficients as Mellin transforms of Bessel functions; Towards a general 
stability, IMRN, 2002:39 (2002), pp. 2075-2119.

[94] F. Shahidi, Functoriality and small eigenvalues of Laplacian on Riemann surfaces, Surveys in Differential Geometry, International Press, to appear.

[95] J. Shalika, The multiplicity one theorem for GL(n), Ann. of Math., 100 (1974), pp. 171-193.

[96] G. Shimura, Introduction to the Arithmetic Theory of Automorphic Functions, Princeton University Press, 1994.

[97] A. Silberger, The Langlands quotient theorem for p-adic groups, Math. Ann., 236 (1978), pp. $95-104$.

[98] D. Soudry, On Langlands functoriality from classical groups to $G L_{n}$, Asterisque, to appear.

[99] J. TAte, Number theoretic background, Proc. Sympos. Pure Math., 33, Part 2 (1979), pp. $3-26$.

[100] D. VoGan, Gelfand-Kirilov dimension for Harish-Chandra modules, Invent. Math., 48 (1997), pp. 75-98. 
F. SHAHIDI 